\title{
Cost-efficient IQ imbalance compensation scheme for DRM plus
}

\author{
Hyun Yang ${ }^{1}$, Kwang-Soo Jeong ${ }^{2}$, Jae-Hoon Yi ${ }^{2}$, Taewon Hwang ${ }^{3}$, \\ and Young-Hwan You ${ }^{1 \mathrm{a})}$ \\ ${ }^{1}$ uT Communication Research Institute, Sejong University, Korea \\ ${ }^{2}$ YuJeong Systems Co. Ltd., Korea \\ ${ }^{3}$ School of Electrical and Electronic Engineering, Yonsei University, Korea \\ a)yhyou@sejong.ac.kr
}

Abstract: In this paper, we propose a low-cost compensation scheme for IQ imbalance using only one pilot symbol for an orthogonal frequency division multiplexing (OFDM) based digital radio mondiale (DRM) system, planned to operate in FM bands. Since the existing methods require more than one OFDM symbol, this scheme is more efficient in computation, yet performs as well as the existing scheme.

Keywords: OFDM, digital radio mondiale, IQ imbalance, single pilot Classification: Science and engineering for electronics

\section{References}

[1] A. Steil, F. Schad, M. Feilen, and E. Hedrich, "Leaving the dead-end street: New way for the digitisation of the VHF-FM sound broadcasting with DRM+," 9th Workshop Digital Broadcasting, Sept. 2008.

[2] A. Tarighat, R. Bagheri, and A. H. Sayed, "Compensation and performance analaysis of IQ imbalance in OFDM receivers," IEEE Trans. Signal Process., vol. 53, no. 8, pp. 3257-3268, Aug. 2005.

[3] J. Tubbax, B. Come, L. Van der Perre, S. Donnay, M. Engels, Hugo De Man, and M. Moonen, "Compensation of IQ imbalance and phase noise in OFDM systems," IEEE Trans. Wireless Commun., vol. 4, no. 5, pp. 872-877, May 2005.

[4] G. Xing, M. Shen, and H. Liu, "Frequency offset and I/Q imbalance compensation for direct-conversion receivers," IEEE Trans. Wireless Commun., vol. 4, no. 2, pp. 673-680, March 2005.

[5] Y. Egashira, Y. Tanabe, and K. Sato, "A novel IQ imbalance compensation method with pilot-signals for OFDM system," VTC-2006 Fall, pp. 1-5, Sept. 2006.

[6] Petition for rulemaking to the united states federal communications commission for in-band on-channel digital audio broadcasting, USADR, Oct. 1998. 


\section{Introduction}

The digital radio mondiale (DRM) consortium has recently proposed to add a new VHF mode to existing DRM modes with a basic $100 \mathrm{KHz}$ bandwidth (BW) for the DRM plus (or DRM robustness mode E), which is used on FM frequency up to $120 \mathrm{MHz}$ [1]. In this mode, BW of approximately $100 \mathrm{KHz}$ is required to provide compatibility with FM broadcasting service. This is small compared to the coherence BW of the channels which is in the order of $1 \mathrm{MHz}$ e.g. for typical urban environments.

DRM plus is an orthogonal frequency division multiplexing (OFDM) based transmission system, which is designed to be compatible with existing FM broadcast band plans. OFDM has been chosen for other broadcasting systems, such as digital audio broadcasting (DAB) and digital video broadcasting (DVB) because of its effective reception capability when dealing with various types of channel impairment. However, IQ imbalance, caused by non-ideal radio frequency $(\mathrm{RF})$ front-ends, can severely degrade system performance [2]. The effects of IQ imbalance on OFDM systems have been investigated [2] and several schemes have been proposed to compensate for IQ imbalance $[3,4,5]$. In [4] and [5], two or more pilot symbols are used to compensate for IQ imbalance.

This paper proposes a low-complexity scheme for correcting IQ mismatch using only one pilot symbol for OFDM-based DRM plus. Because the channel is usually frequency-flat for FM broadcasting applications, utilization of this knowledge enables a low-complexity implementation of the IQ imbalance compensation scheme. The performance of the proposed algorithm is demonstrated via several examples in the channel models for FM bands.

\section{Signal description}

We consider an OFDM system with $N$ subcarriers and $N_{g}$ samples for guard interval (GI). The OFDM system can simultaneously transmit $N$ symbols $\left\{X_{l}(k)\right\}_{k=0}^{N-1}$ during the $l$-th period, which consists of $N_{p}$ pilot symbols and $N-N_{p}$ data symbols.

Denote $x_{l}(n)$ and $h_{l}(n)$ to be the transmitted complex signal after IFFT during the $l$-th period in time domain and channel impulse response, respectively. After removing the cyclic prefix and assuming perfect IQ balance, the received signal can be written as

$$
y_{l}(n)=h_{l}(n) \otimes x_{l}(n)+w_{l}(n),
$$

where $\otimes$ and $w_{l}(n)$ denote circular convolution and zero-mean complex white Gaussian noise with variance $\sigma_{n}^{2}$, respectively. For IQ mismatch, on the other hand, the received signal is expressed in [3]

$$
y_{l}(n)=u r_{l}(n)+v r_{l}^{*}(n)+w_{l}(n),
$$

where $r_{l}(n)=h_{l}(n) \otimes x_{l}(n), u$ and $v$ are IQ imbalance parameters. The $u$ and $v$ reflect the effect of IQ imbalance as in [5]

$$
u=\frac{1+\varepsilon \cdot \exp (-j \Delta \phi)}{2}, \quad v=\frac{1-\varepsilon \cdot \exp (j \Delta \phi)}{2},
$$


where $\varepsilon$ and $\Delta \phi$ are the amplitude and phase imbalances between the inphase and quadrature components, respectively.

After FFT in (2), the received OFDM symbol in the frequency domain can be written as

$$
Y_{l}(k)=u H_{l}(k) X_{l}(k)+v H_{l}^{*}(N-k) X_{l}^{*}(N-k)+W_{l}(k), 1 \leq k \leq N-1
$$

where $H_{l}(k)$ is the channel frequency response and $W_{l}(k)$ is the additive white Gaussian noise during the $l$-th period. We assume perfect synchronization and concentrate on the IQ mismatch problem.

\section{Low-complexity 10 compensation scheme for DRM plus}

In this section, we derive a correction scheme for IQ imbalance using the pilot subcarriers in one OFDM symbol. For simplicity of the algorithm, the proposed method employs gain reference pilot subcarriers defined in DRM systems, which are symmetrically distributed around the DC subcarrier.

\subsection{Algorithm description}

In this section, we omit noise $W_{l}(k)$ for simplicity. Consider the received pilot symbol at the $k_{j}$-th subcarrier

$$
Y_{l}\left(k_{j}\right)=u H_{l}\left(k_{j}\right) X_{l}\left(k_{j}\right)+v H_{l}^{*}\left(N-k_{j}\right) X_{l}^{*}\left(N-k_{j}\right)
$$

for $j=1,2, \ldots, N_{p}$. We add together the first $N_{p} / 2$ and then the last $N_{p} / 2$ pilot symbols for the received OFDM symbol as follows:

$$
\begin{aligned}
\sum_{j=1}^{N_{p} / 2} Y_{l}\left(k_{j}\right) & =u \sum_{j=1}^{N_{p} / 2} H_{l}\left(k_{j}\right) X_{l}\left(k_{j}\right)+v \sum_{j=1}^{N_{p} / 2} H_{l}^{*}\left(N-k_{j}\right) X_{l}^{*}\left(N-k_{j}\right) \\
& =\sum_{j=1}^{N_{p} / 2} \alpha X_{l}\left(k_{j}\right)+\sum_{j=N_{p} / 2+1}^{N_{p}} \beta X_{l}^{*}\left(k_{j}\right)
\end{aligned}
$$

and

$$
\begin{aligned}
\sum_{j=N_{p} / 2+1}^{N_{p}} Y_{l}\left(k_{j}\right) & =u \sum_{j=N_{p} / 2+1}^{N_{p}} H_{l}\left(k_{j}\right) X_{l}\left(k_{j}\right) \\
& +v \sum_{j=N_{p} / 2+1}^{N_{p}} H_{l}^{*}\left(N-k_{j}\right) X_{l}^{*}\left(N-k_{j}\right) \\
& =\sum_{j=N_{p} / 2+1}^{N_{p}} \alpha X_{l}\left(k_{j}\right)+\sum_{j=1}^{N_{p} / 2} \beta X_{l}^{*}\left(k_{j}\right),
\end{aligned}
$$

where $\alpha$ and $\beta$ are IQ imbalance factors incorporating the frequency-flat channel response. 
From (6)-(7), the estimate of $\hat{\alpha}$ and $\hat{\beta}$ can be obtained by using the known pilot symbols for the receiver:

$$
\hat{\alpha}=\frac{C_{l, 0}^{*} \sum_{j=1}^{N_{p} / 2} Y_{l}\left(k_{j}\right)-C_{l, 1}^{*} \sum_{j=N_{p} / 2+1}^{N_{p}} Y_{l}\left(k_{j}\right)}{\left|C_{l, 0}\right|^{2}-\left|C_{l, 1}\right|^{2}}
$$

and

$$
\hat{\beta}=\frac{C_{l, 0} \sum_{j=N_{p} / 2+1}^{N_{p}} Y_{l}\left(k_{j}\right)-C_{l, 1} \sum_{j=1}^{N_{p} / 2} Y_{l}\left(k_{j}\right)}{\left|C_{l, 0}\right|^{2}-\left|C_{l, 1}\right|^{2}}
$$

where

$$
C_{l, 0}=\sum_{j=1}^{N_{p} / 2} X_{l}\left(k_{j}\right), \quad C_{l, 1}=\sum_{j=N_{p} / 2+1}^{N_{p}} X_{l}\left(k_{j}\right) .
$$

In (10), the pilot symbol must be selected so that the denominators in (8)-(9) are nonzero, i.e., $\left|C_{l, 0}\right|^{2}-\left|C_{l, 1}\right|^{2} \neq 0$. Note that $C_{l, m} /\left(\left|C_{l, 0}\right|^{2}-\left|C_{l, 1}\right|^{2}\right)$ and $C_{l, m}^{*} /\left(\left|C_{l, 0}\right|^{2}-\left|C_{l, 1}\right|^{2}\right)(m=0,1)$ are known at the receiver.

From the parameters obtained above, the compensation scheme for IQimbalance can be described by

$$
\hat{X}_{l}(k)=\frac{\hat{\alpha}^{*} Y_{l}(k)-\hat{\beta} Y_{l}^{*}(N-k)}{|\hat{\alpha}|^{2}-|\hat{\beta}|^{2}}, 1 \leq k \leq N-1
$$

where $\hat{X}_{l}(k)$ is the estimate of $X_{l}(k)$.

\subsection{Complexity}

From the computations above, the proposed scheme uses sums over $N_{p}$ pilot subcarriers for estimating $\alpha$ and $\beta$. However, the conventional method in [5] uses products of $2 N_{p}$ pilots contained in two OFDM symbols. In the conventional scheme, the estimate of $\hat{\alpha}$ and $\hat{\beta}$ becomes:

$$
\hat{\alpha}=\frac{1}{N_{p}} \sum_{j=1}^{N_{p}} \frac{X_{l+D}^{*}\left(N-k_{j}\right) Y_{l}\left(k_{j}\right)-X_{l}^{*}\left(N-k_{j}\right) Y_{l+D}\left(k_{j}\right)}{\Delta\left(k_{j}\right)}
$$

and

$$
\hat{\beta}=\frac{1}{N_{p}} \sum_{j=1}^{N_{p}} \frac{X_{l}^{*}\left(k_{j}\right) Y_{l+D}\left(k_{j}\right)-X_{l+D}^{*}\left(k_{j}\right) Y_{l}\left(k_{j}\right)}{\Delta\left(k_{j}\right)}
$$

where $\Delta\left(k_{j}\right)=X_{l}\left(k_{j}\right) X_{l+D}^{*}\left(N-k_{j}\right)-X_{l}^{*}\left(N-k_{j}\right) X_{l+D}\left(k_{j}\right) \neq 0$ and $D$ represents the distance between two pilot symbols.

By assuming that $X_{l+m}^{*}\left(N-k_{j}\right) / \Delta\left(k_{j}\right)$ and $X_{l+m}^{*}\left(k_{j}\right) / \Delta\left(k_{j}\right)(m=0, D)$ in (12) and (13) are known at the receiver, one can find that the conventional scheme requires $4 N_{p}$ complex multiplications and $4 N_{p}-2$ complex additions. On the other hand, the proposed method requires four complex multiplications and $N_{p}$ complex additions to obtain (8) and (9). Therefore, the proposed method can be implemented with less complexity than the conventional scheme because the number of complex multiplications and additions are reduced by a factor of $N_{p}$ and 4 , respectively. 
Table I. Channel profiles

\begin{tabular}{|c|c|c|c|c|}
\hline No & Channel model & Velocity & no. of path & Maximum delay \\
\hline \hline 1 & Urban (slow) & $2 \mathrm{~km} / \mathrm{h}$ & 9 & $3 \mu \mathrm{s}$ \\
\hline 2 & Urban (fast) & $60 \mathrm{~km} / \mathrm{h}$ & 9 & $3 \mu \mathrm{s}$ \\
\hline 3 & Rural & $150 \mathrm{~km} / \mathrm{h}$ & 9 & $3 \mu \mathrm{s}$ \\
\hline 4 & Terrain obstructed & $60 \mathrm{~km} / \mathrm{h}$ & 9 & $16 \mu \mathrm{s}$ \\
\hline 5 & Hilly terrain & $100 \mathrm{~km} / \mathrm{h}$ & 12 & $84.5 \mu \mathrm{s}$ \\
\hline
\end{tabular}

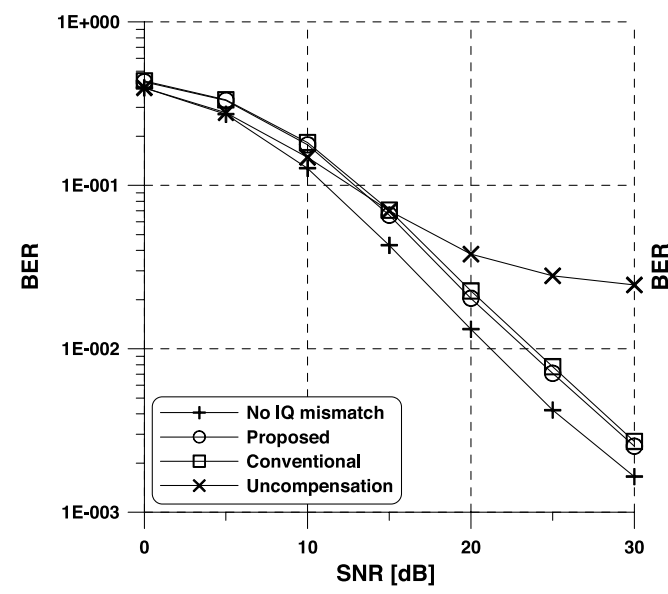

(a)

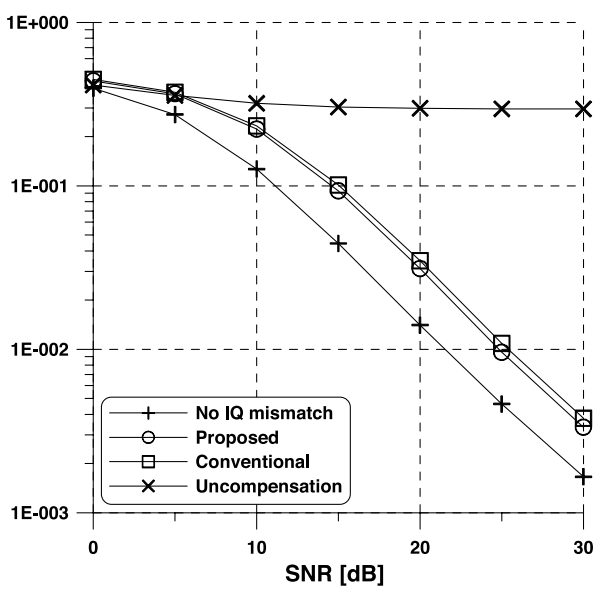

(b)

Fig. 1. Performance of the IQ compensation schemes for CM1: (a) $\varepsilon=0.1 \mathrm{~dB}$ and $\Delta \phi=10^{\circ}$ (b) $\varepsilon=0.5 \mathrm{~dB}$ and $\Delta \phi=50^{\circ}$

\section{Simulation results}

In this section, we verify the effectiveness of the proposed method through simulation. System parameters were chosen according to [1] as follows: $N=$ 213, $N_{g}=21, N_{p}=14$, and 64QAM at carrier frequency $90 \mathrm{MHz}$ with $95 \mathrm{KHz}$ bandwidth. The channel models (CMs) 1 5 were based on an EIA channel model [6], which is widely used in the performance analysis of FM and is summarized in Table I.

In addition, performance was compared with the conventional method [5]. For fair comparison, the $N_{p}$ pilot subcarriers chosen from two consecutive OFDM symbols were used for the conventional method, i.e. $D=1$ in (12) and (13). Here, the pilot symbol of the conventional method was designed by [5] and the proposed method employed a gain reference pilot.

Figure 1 demonstrates the BER performances with respect to $\varepsilon$ and $\Delta \phi$ when CM1 is used. If left uncompensated, even small amounts of IQ mismatch result in severe performance degradation. The performance of the proposed scheme is shown to be close to that of the conventional scheme, while the computational cost of the proposed method is shown to be significantly reduced, as confirmed in Section 3.2.

Figure 2 shows the BER performances for CM2 and CM3 when $\varepsilon=$ 0.5 and $\Delta \phi=50^{\circ}$. From this figure, we observe that the increase in time 


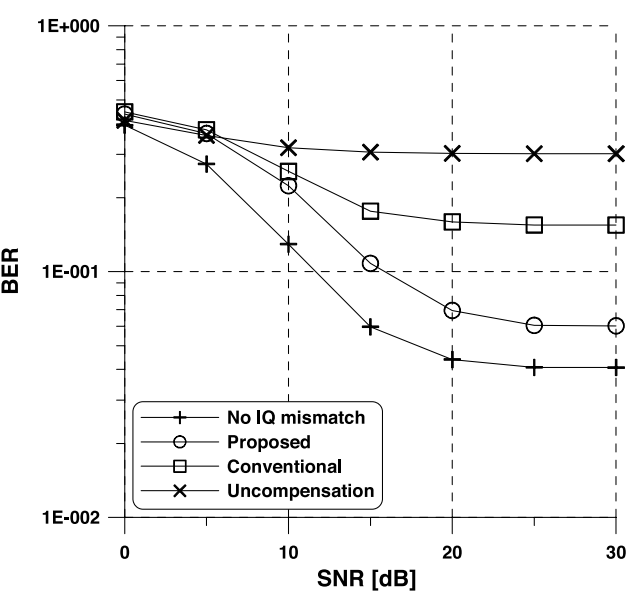

(a) $\mathrm{CM} 2$

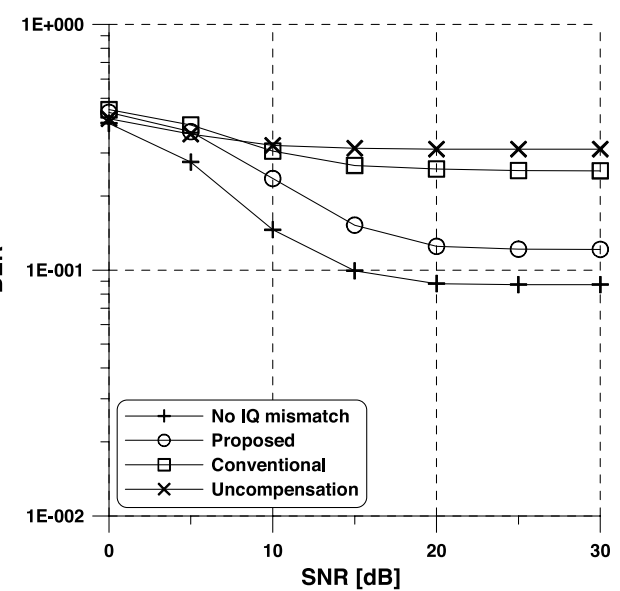

(b) $\mathrm{CM} 3$

Fig. 2. Performance of the IQ compensation schemes for $\varepsilon=0.5 \mathrm{~dB}$ and $\Delta \phi=50^{\circ}$

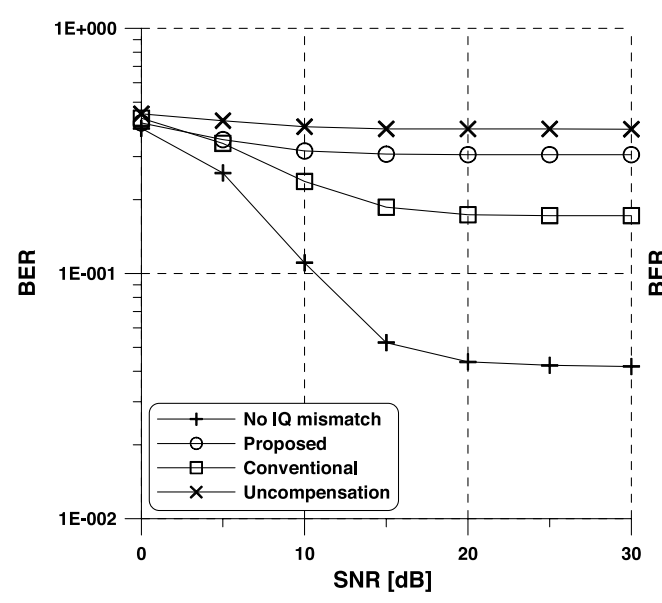

(a) $\mathrm{CM} 4$

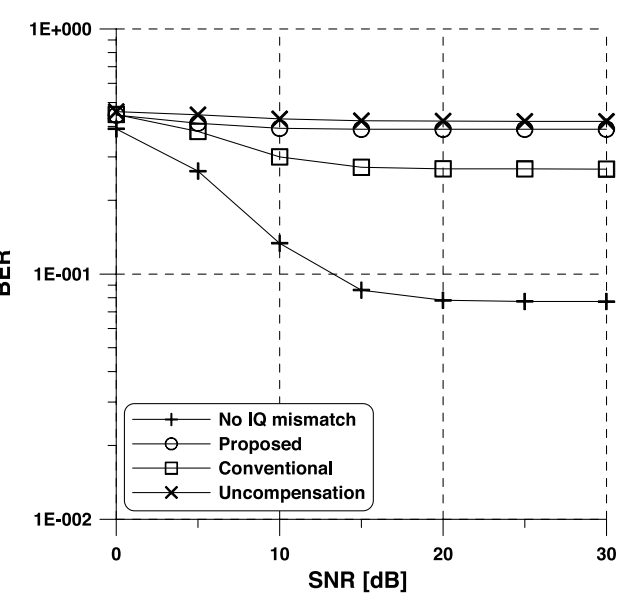

(b) CM5

Fig. 3. Performance of the IQ compensation schemes for $\varepsilon=0.5 \mathrm{~dB}$ and $\Delta \phi=50^{\circ}$

selectivity, as in CM2 and CM3, degrades the performance of the conventional scheme, whereas the performance of the proposed scheme is less affected by time selectivity because it uses one pilot symbol.

The BER performance in CM4 and CM5 is also depicted in Fig. 3 when $\varepsilon=0.5$ and $\Delta \phi=50^{\circ}$. This figure shows that the performance of the proposed scheme declines with an increase in the amount of delay spread of the channel, which primarily stems from the increased frequency selectivity.

\section{Conclusion}

We introduced a simple compensation scheme for IQ imbalance for digital FM broadcasting applications. This scheme can efficiently correct IQ imbalance using one pilot symbol. The proposed scheme was computationally efficient with performance comparable to the existing estimator. 


\section{Acknowledgment}

The authors wish to acknowledge the assistance and support of the Center for Advanced Transceiver Systems and the Ministry of Knowledge Economy, and this research is supported by Seoul R\&BD Program. 